Computer Applications: An International Journal (CAIJ), Vol.3, No.1, February 2016

\title{
ZINC SUPPLEMENTATION MAY REDUCE THE RISK OF HEPATOCELLULAR CARCINOMA USING BIG DATA NETWORK ANALYSIS
}

\author{
Jinyu $\mathrm{Hu}^{1,2, \dagger}$, Bin $\mathrm{Hu}^{1, \dagger}$, Weisen $\mathrm{Pan}^{3}$, Junsong Wang ${ }^{4}$, Xiangtao Liu ${ }^{2}$, Zhiwei \\ $\mathrm{Gao}^{5^{*}}$ \\ ${ }^{1}$ Division of Immunology and Rheumatology, Department of Medicine, Stanford \\ University, Stanford, CA, USA 94305; and Department of Medicine, Veterans Affairs \\ Palo Alto Health Care System, Palo Alto, CA, USA 94306 \\ ${ }^{2}$ Cipher Ground , 675 Rt 1 South North Brunswick, NJ, USA 08902 \\ ${ }^{3}$ Department of Computer Science and Engineering, The Ohio State University, \\ Columbus, OH, USA 43210 \\ ${ }^{4}$ School of Biomedical Engineering, Tianjin Medical University, Tianjin 300072, China \\ ${ }^{5}$ School of Computing, Engineering and Information Sciences, Northumbria University, \\ Newcastle upon Tyne, NE1 8ST, United Kingdom
}

\begin{abstract}
Hepatocellular carcinoma $(H C C)$ is a primary liver cancer with poor survival rates. Gene expression data of HCC are investigated to screen target genes and core genes, which are employed to propose a new strategy for the treatment of HCC. New concepts such as gene data streams, gene characteristic strength $(C S)$, gene impact factor $(G I F)$ and gene force $(G F)$ are proposed. Together with gene community network $(G C N)$, a novel algorithm, that is, called gene force algorithm (GFA), is presented to screen feature genes, target genes and core genes. The fifteen target genes are obtained, which can be divided into three clustering sets including HAMP Cluster $=\{H A M P$, Trans, AQP4,VIPR1 $\}$, MT Cluster $=\{M T 1 H, M T 1 B$, MTIG, MT1E, MTIL, RNAHP, DNASE1L3 $\}$ and GPC3 Cluster $=\{G P C 3\}$. The core genes of each clusters are HAMP, Metallothionein genes (MTs) and GPC3 respectively, where MTs is a general name for a group of metallothionein genes. According to the relationship between the three core genes and the metals including copper, iron and zinc, a treatment strategy for HCC is proposed, namely, "Supplement Zinc after surgery" for HCC patients. The proposed treatment method can be used to regulate the expression levels of HCC core genes.
\end{abstract}

\section{KEYWORDS}

Core gene, gene community network, gene regulation, HCC, zinc supplementation

\section{INTRODUCTION}

Hepatocellular carcinoma (HCC) is the third leading cause of the world-cancer-related death. [1]. It is vital to develop efficient and safe treatments of HCC for clinical services in order to save or prolong patients' lives. It has been recognized that gene therapy is one of the most promising solutions to the treatments of HCC.

The traditional feature genes approaches only consider the differences of the gene expressions between tumour and normal tissues [2]; however, the degrees of the differences are not taken into 
Computer Applications: An International Journal (CAIJ), Vol.3, No.1, February 2016

account. The intensity of the correlations between genes has not been clearly defined and interpreted. The absolute values of Pearson's correlation coefficients are usually used to assess the correlation of genes [3], which fail to distinguish the positive correlation from the negative correlation. Target genes are usually obtained by using biological experiments [4], but it is noted that the experiments are difficult to implement and the coverage is often small. There seems not to be an agreed opinion on the reason caused tumour. One opinion is that oncogenes being active and anti-oncogenes being inactive will cause tumour [5]. Another one emphasizes that the mutation of the oncogenes and anti-oncogenes will lead to cancer [6]. Recently, gene therapy has been applied in gene replacement, gene correction, gene augmentation, and gene inactivation [7]. Unfortunately, the conventional gene therapy methods [8] seem to be difficult to implement and their effectiveness has yet proved significantly.

Currently, the big data [9-16] study is overwhelming, we can consider using the network method to solve biological data. As a result, there is a strong motivation to investigate gene therapy from the viewpoint of big data, providing a new idea for the treatment of HCC. In this study, the gene community network (GCN) proposed in [17-21] can be divided into gene positive network (GPN or POS network) and gene negative network (GNN or NEG network), which are employed here to describe the facilitating impact between the same type genes and the restraining impact between the opposite type genes. The gene characteristic strength (CS) and the CS threshold (CST) are then defined, from which the genes with CS values being greater than the CST are described as feature genes. The genes impact factor (GIF) is next defined as the sum of all the Pearson correlation strength between this gene and all other genes. The gene force (GF) is defined as the multiplication of CS and GIF. Together with the GCN, the concepts of the gene data stream, CS, GIF and GF pave the way for developing a novel algorithm, called GF algorithm (GFA), in order to screen the target genes and core genes. Finally, a simple and safe method is presented to regulate gene expression levels, providing a new insight into the treatment of HCC.

\section{MATERIAL AND METHODS}

\subsection{Data}

The HCC data are available from the NCBI database GSE3500[22]. There are 3964 genes with different expression in 156 liver tissues (74 non-tumour liver tissues and $82 \mathrm{HCC}$ tissues).

\subsection{Basic concept}

In order to develop an algorithm to screen target genes, a number of important concepts such as gene community network, gene characteristic strength, gene impact factor, and gene forces are proposed and discussed.

A Gene Force(GF) is determined by both the total impacts between the characteristics strength (CS) and the gene impact factor (GIF). The greater the gene force, it is more likely for this gene to become a target gene. The GF is defined as the multiplication of the $\mathrm{CS}$ and GIF, that is,

$\mathrm{G} F=\mathrm{ICS} * \mathrm{GIF}$

Where $\mathrm{GIF}=\sum\left|\mathrm{p}_{\mathrm{ij}}\right|$, and the absolute value of $p_{i j}$, which is the Pearson correlation between gene $i$ and gene $j$, is greater 0.2 , and $C S=\mid$ mean(Tumor) - mean(Normal) $\mid$. 


\subsection{Core gene algorithm (CGA)}

Many different HCC target genes can be obtained from different angles or different method. But it is difficult to adopt measures for each target gene. They follow the leader, we mainly directed against the most influential of these target genes, which called core genes. And the algorithm steps (see Figure 2) are as follows:

(a) The feature genes are selected from the original gene.

After Replacing missing values and calculating the CS value of all original genes, the $\mathrm{K}$ genes of the largest CS values as feature genes. In this paper, $\mathrm{K}=100$.

(b) The candidate target genes are choosing from feature genes.

From the feature genes, select the genes which rather than the CS mean value as a candidate target gene.

(c) The target genes are screened from the candidate target genes.

The target genes, which CS value are greater than the GF mean value, are screened in the features genes.

(d) Finally, the core genes are picked from the target genes.

The GCN network is built based on the target genes, and the core genes can be gotten after analysis of the network.

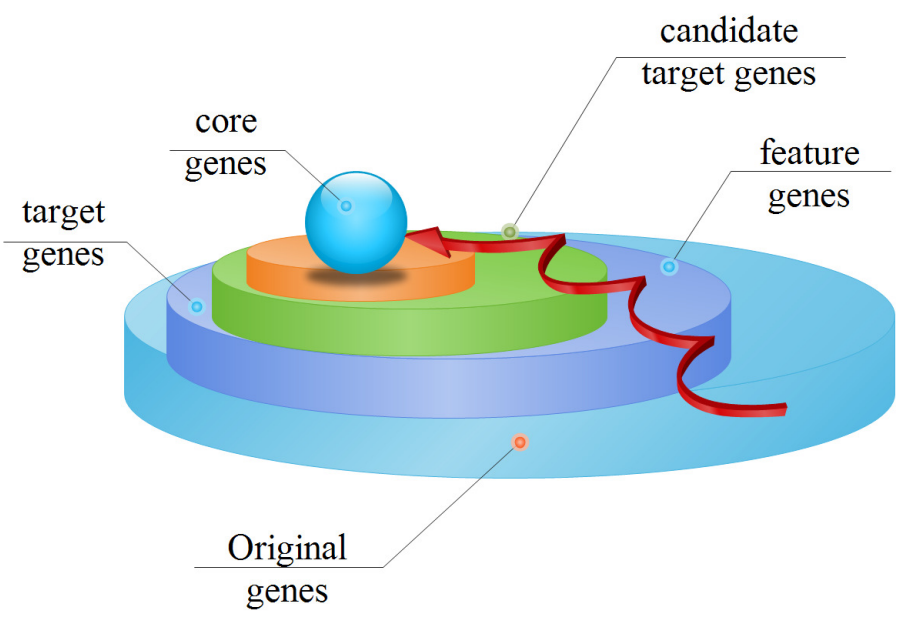

Figure 1. The relationship of five categories of genes.

\section{RESULTS}

\subsection{HCC target genes}

Based on the CGS algorithm proposed, we can screen HCC target genes following the steps:

(a) Calculate the CS values of the original genes, and sort the genes in descending order according to the CS values. One can obtain the top $100(\mathrm{~K}=100)$ genes with larger CS values. 
Computer Applications: An International Journal (CAIJ), Vol.3, No.1, February 2016

(b) The CS average value of the top 100 genes is 2.37 , which is set as the threshold CST $=2.37$, leading to $30(\mathrm{R}=30)$ candidate target genes with $\mathrm{CS}$ values larger than CST.

(c)The average GF value of the 30 candidate target genes obtained is calculated as 129.68 , which is set as the threshold GFT=129.68. We thus can obtain the $13(\mathrm{Q}=13)$ target genes, described by table 1 whose GF values are larger than GFT.

Table 1. The target genes

\begin{tabular}{|c|c|c|c|c|}
\hline GID & GName & CS & GIF & GF \\
\hline GENE7X & HAMP & 38.75 & 12.91 & 500.1 \\
\hline GENE86X & MT1H & 13.24 & 17.58 & 232.71 \\
\hline GENE531X & RNAHP & 11.15 & 17.7 & 197.3 \\
\hline GENE3131X & MT1G & 12.7 & 15.54 & 197.3 \\
\hline GENE1288X & RNAHP & 13.71 & 13.91 & 190.66 \\
\hline GENE3579X & GPC3 & 18.3 & 10.19 & 186.46 \\
\hline GENE2088X & MT1L & 10.64 & 16.1 & 171.31 \\
\hline GENE414X & Trans & 10.1 & 15.64 & 157.98 \\
\hline GENE3558X & AQP4 & 10.81 & 14.08 & 152.2 \\
\hline GENE1911X & Trans & 9.46 & 16.05 & 151.85 \\
\hline GENE676X & ADH4 & 9.53 & 14.51 & 138.24 \\
\hline GENE244X & MT1E & 8.95 & 15.42 & 138.03 \\
\hline GENE976X & VIPR1 & 7.79 & 17.19 & 133.91 \\
\hline
\end{tabular}

Trans: Transcribed locus; the duplicate genes are the same ones.

In Table 1, the 13 target genes are shown with names, and the values of CS, GIF and GF. If the Transcribedlocus and the duplication of genes are extracted, the set of the target genes can be given by TGS $=\{$ HAMP, MT1H, RNAHP, MT1G, RNAHP, GPC3, MT1L, AQP4, ADH4, MT1E, VIPR1\}, where the genes are listed in descending order in terms of GF values.

\subsection{HCC core genes}

On the basis of the 13 target genes, we can construct the GCN networks for further analysis in order to screen the most important HCC target genes, called HCC core genes. Firstly, we calculate the Pearson correlation of target genes (see table 2). 
Computer Applications: An International Journal (CAIJ), Vol.3, No.1, February 2016

Table 2. Pearson correlation of the target genes

\begin{tabular}{|c|c|c|c|c|c|c|c|c|c|c|c|c|c|}
\hline & GPC3 & Trans & Trans & HAMP & AQP4 & MTlL & MTlH & RNAHP & RNAHP & MTlG & MTlE & VIPRl & ADH4 \\
\hline GPC3 & 1.00 & -0.35 & -0.33 & -0.29 & -0.31 & -0.40 & -0.38 & -0.37 & -0.26 & -0.34 & -0.35 & -0.42 & -0.37 \\
\hline Trans & -0.35 & 1.00 & 0.84 & 0.76 & 0.81 & 0.63 & 0.76 & 0.75 & 0.59 & 0.61 & 0.66 & 0.73 & 0.49 \\
\hline Transc & -0.33 & 0.84 & 1.00 & 0.63 & 0.66 & 0.59 & 0.71 & 0.75 & 0.55 & 0.71 & 0.61 & 0.70 & 0.51 \\
\hline HAMP & -0.29 & 0.76 & 0.63 & 1.00 & 0.89 & 0.47 & 0.54 & 0.51 & 0.42 & 0.49 & 0.44 & 0.53 & 0.37 \\
\hline AQP4 & -0.31 & 0.81 & 0.66 & 0.89 & 1.00 & 0.54 & 0.62 & 0.58 & 0.52 & 0.51 & 0.56 & 0.59 & 0.38 \\
\hline MTlL & -0.40 & 0.63 & 0.59 & 0.47 & 0.54 & 1.00 & 0.84 & 0.85 & 0.79 & 0.69 & 0.83 & 0.67 & 0.50 \\
\hline MTlH & -0.38 & 0.76 & 0.71 & 0.54 & 0.62 & 0.84 & 1.00 & 0.95 & 0.77 & 0.77 & 0.83 & 0.76 & 0.61 \\
\hline RNAHP & -0.37 & 0.75 & 0.75 & 0.51 & 0.58 & 0.85 & 0.95 & 1.00 & 0.80 & 0.75 & 0.83 & 0.77 & 0.60 \\
\hline RNAHP & -0.26 & 0.59 & 0.55 & 0.42 & 0.52 & 0.79 & 0.77 & 0.80 & 1.00 & 0.55 & 0.74 & 0.51 & 0.35 \\
\hline MT1G & -0.34 & 0.61 & 0.71 & 0.49 & 0.51 & 0.69 & 0.77 & 0.75 & 0.55 & 1.00 & 0.66 & 0.62 & 0.49 \\
\hline MT1E & -0.35 & 0.66 & 0.61 & 0.44 & 0.56 & 0.83 & 0.83 & 0.83 & 0.74 & 0.66 & 1.00 & 0.63 & 0.45 \\
\hline VIPR1 & -0.42 & 0.73 & 0.70 & 0.53 & 0.59 & 0.67 & 0.76 & 0.77 & 0.51 & 0.62 & 0.63 & 1.00 & 0.68 \\
\hline ADH4 & -0.37 & 0.49 & 0.51 & 0.37 & 0.38 & 0.50 & 0.61 & 0.60 & 0.35 & 0.49 & 0.45 & 0.68 & 1.00 \\
\hline
\end{tabular}

The correlation matrix is denoted by P. To cluster the target genes, we set different threshold values. The thresholds were obtained by the methods as follows:

(1) the positive threshold "T1" <=the positive minimum of maximum values in each column "mm1". In this paper, the $\mathrm{T} 1=\left[\begin{array}{llllllll}0.6787 & 0.7688 & 0.8047 & 0.8339 & 0.8434 & 0.8923 & 0.9998 & 0.9998\end{array}\right.$ $\left.\begin{array}{lllll}0.9998 & 0.9998 & 0.9998 & 0.9998 & 0.9998\end{array}\right]$

(2) the negative threshold "T2" $<=$ the negative maximum of maximum values in each column

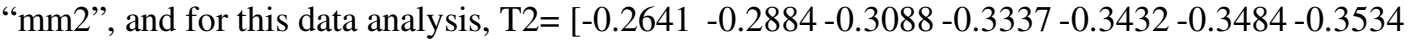
$-0.3693-0.3723-0.3781-0.403-0.4177-0.9999]$

Three threshold values were chosen for clustering, that is : $-0.2641,0.6787,0.7688$. Based on different threshold, we get different GCN Networks (see Figure 2).

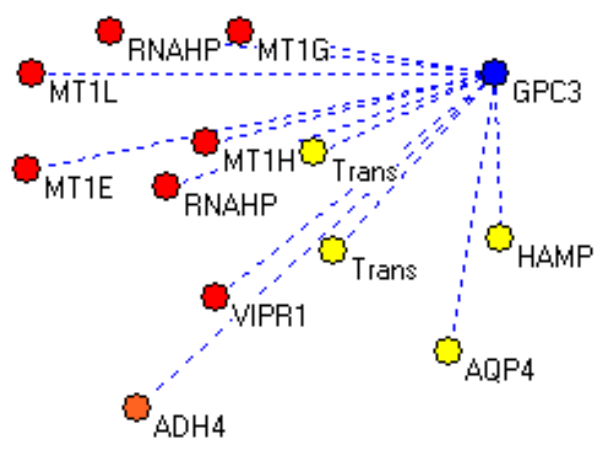

(a)GNN network $(\mathrm{T}=-0.2641)$ 
Computer Applications: An International Journal (CAIJ), Vol.3, No.1, February 2016

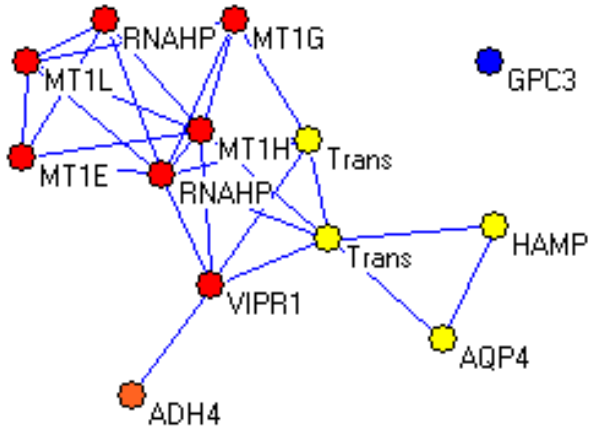

(b)GPN network ( $\mathrm{T}=0.6787$ )

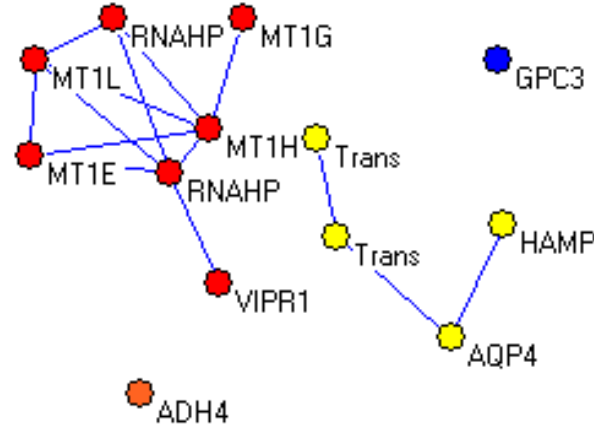

(c) GPN network $(\mathrm{T}=0.7688)$

Figure 2.GCN networks of target genes.

In the figure 2, (a) when T=-0.2642, the GPC3 is negative related to other genes; (b) when $\mathrm{T}=0.6787$, we get a big cluster $\{$ HAMP, Trans, Trans, AQP4, MT1H, MT1B, MT1G, MT1E, MTIL, RNAHP, VIPR1, ADH4\}. (c) when $\mathrm{T}=0.7688$, the big cluster is divided to two clusters \{HAMP, Trans, Trans, AQP4 $\}$ and $\{$ MT1H, MT1B, MT1G, MT1E, MTIL, RNAHP, VIPR1, ADH4\}.

According the analysis above, the target genes are obtained, which can be divided into three clustering sets (see Figure 3 ) including HAMP Cluster $=\{$ HAMP, Trans, AQP4, VIPR1 $\}$, MT Cluster $=\{$ MT1H, MT1B, MT1G, MT1E, MTIL, RNAHP, DNASE1L3 $\}$ and GPC3 Cluster $=\{$ GPC 3$\}$. The core genes of each clusters are then derived, that is, HAMP, MTs and GPC 3 respectively, where MTs is a general name for a group of metallothionein genes including MT1H, MT1B, MT1G, MT1E, and MTIL.

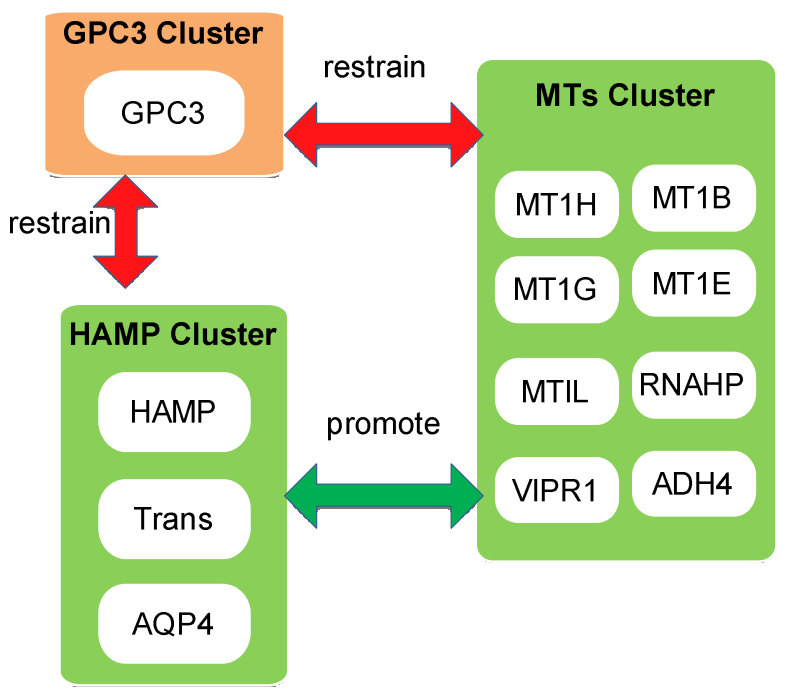

Figure 3 Three clusters and core genes

At last, we get three core genes HAMP, MTs and GPC3. The HAMP genes is to regulate the iron homeostasis in the human body [23], and is the Iron overload brings negative impact for the HCC [24]. And the aquaporin plays a role in the hepatocellular [25]. 
The Metallothionein genes (MTs) is very important role to adjust the heavy metal in the body [26], and the HCC patients with high zinc- Color rate [27]. In addition, the RNAPH [28], which has another name DDX42, is apoptosis in the HCC patients.

And the GPC3 gene is a very significant oncogene, which is over- expressed in the fetal liver [29] and is normal in healthy adults, and it's a new biomarker for live cancer [30].

\section{DISCUSSION}

\subsection{Correlations between oncogenes and anti-oncogenes}

A single gene generally cannot perform a function independently. There is mutual promotion or mutual inhibition relationship between genes. Oncogenes and tumour anti-oncogenes are obviously in opposite types. In order to illustrate the correlations between oncogenes and antioncogenes, we use the top 100 feature genes, including 34 oncogenes and 66 anti-oncogenes (see Figure 3)

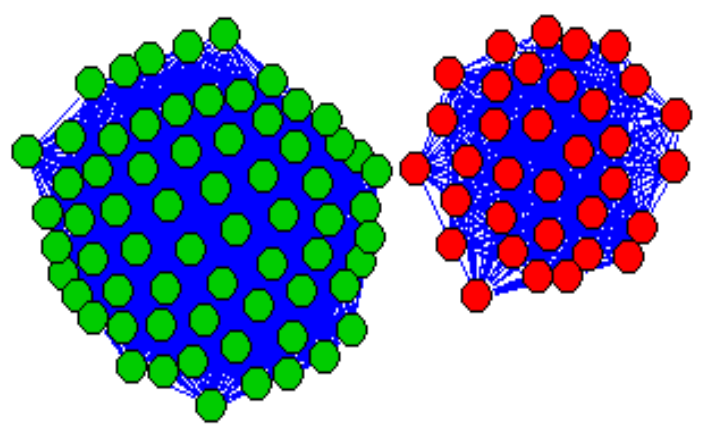

(a) The positive relationship between oncogenes and anti-oncogenes.

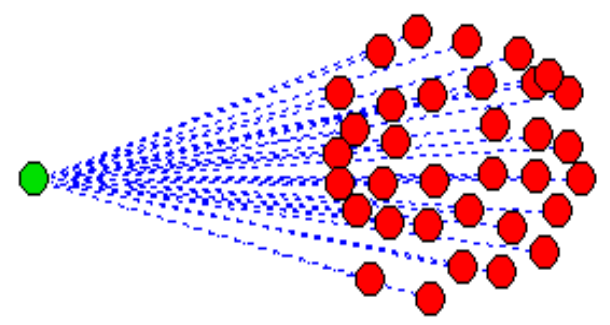

(c). An oncogene and anti-oncogenes have negative relationship

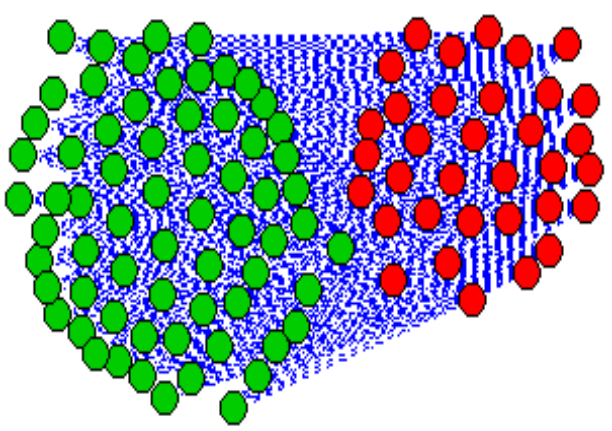

(b) The negative relationship between oncogenes and anti-oncogenes (macro diagram).

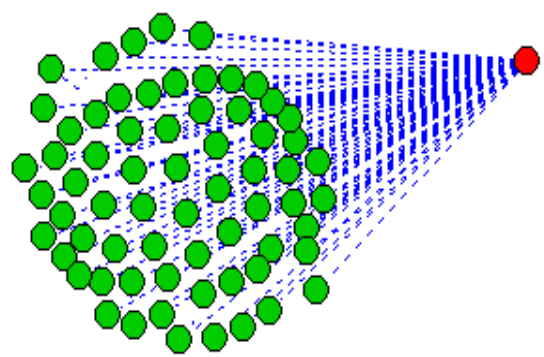

(d). An anti-oncogene and proto-oncogenes have negative relationship

Figure 4. The relationship between oncogenes and anti-oncogenes

In the figure 4, the green nodes represent anti-oncogenes, the yellow nodes represent oncogenes, the solid lines represent positive correlation, and dotted lines represent negative correlation, respectively. The lines with Pearson correlation strength defined by equation (2) being smaller than 0.2 are regarded as noises, which are removed in Figures 3. 
To summarize, one can conclude that: a) Genes in the same type are positively related, indicating a mutual promotion relationship. b) Genes with opposite types are negatively related, which inhibit each other.

\subsection{The importance of zinc supplementation}

The aim of the treatment is to make the core genes GPC3, HAMP and MTs to come back to the normal expression level, that is, we have to do something such that GPC3 $\downarrow$ HAMP $\uparrow$ MTs $\uparrow$. In the same time, we also hope the contents of trace elements such as irons, coopers and zincs to recover to normal content levels. As a result, we have to make $\mathrm{Fe}^{2+} \downarrow \mathrm{Cu}^{2+} \downarrow \mathrm{Zn}^{2+} \uparrow$.

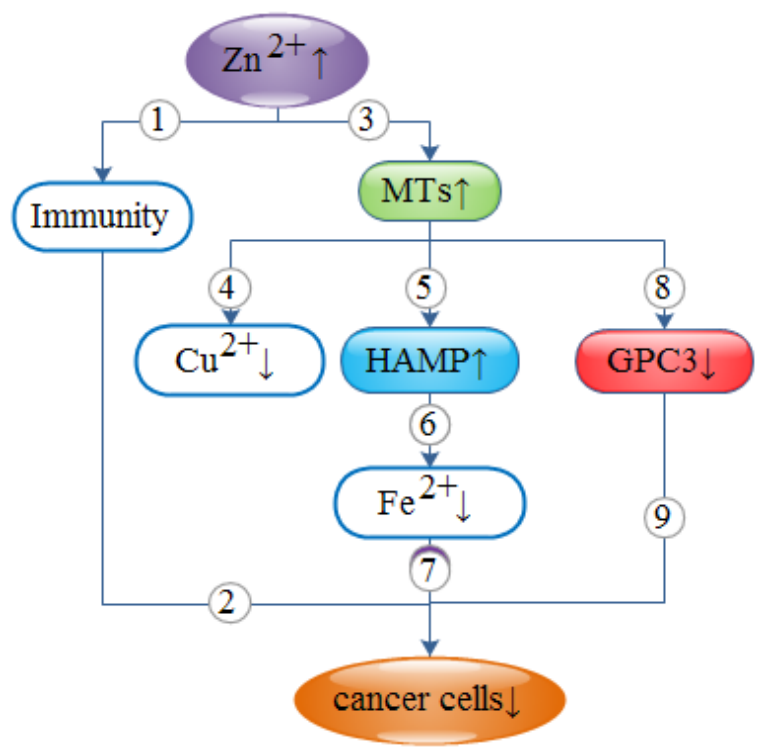

Figure 5. Supplement Zn for HCC patients.

The principle of the zinc supplementation is illustrated as follows (see figure 5) :

i) Zinc deficiency associates with many symptoms of liver cancer patients [31] and $\mathrm{Zn}$ supplementation can improve immunity of patients.

ii) To some degree, the immune system can inhibit cancer cell proliferation.

iii) Adding Zn can make the expression level of MTs up-regulate [32].

iv) The high-level expression of MTs can make metallothionein proteins bond free copper ions, leading to the reduction of the content of free copper ions in serum.

v) MTs and HAMP mutually promote so that the up-regulation of the expression level of MTs will stimulate the up-regulation of the expression level of HAMP.

vi) The up-regulation of the expression level of HAMP will lower the content of irons in the serum. 
Computer Applications: An International Journal (CAIJ), Vol.3, No.1, February 2016

vii) As the reduction of irons in the serum, the transported oxygen is decreased so that the proliferation speed of the cancel cells slow down, and even some cancer cells die due to insufficient supply of oxygen.

viii) MTs and GPC3 inhibit mutually so that the up-regulation of the expression level of the MTs will lead to the down-regulation of the expression level of the GPC3.

ix) The low-level expression of the GPC3 may slow down the proliferation of cancer cells. Papers in this format must not exceed twenty (20) pages in length. Papers should be submitted to the secretary AIRCC. Papers for initial consideration may be submitted in either .doc or .pdf format. Final, camera-ready versions should take into account referees' suggested amendments.

\section{CONCLUSION}

Big data is a new and main trend, and this paper proposes a gene networks to analysis the biology big data. And the article presents result of Hepatocellular carcinoma (HCC), which is a primary malignancy of the liver cancer, as follows:

(1) To bring the liver cancer under control, we should rely mainly on the suppressor genes (e.g. HAMP) supplemented by oncogenes (e.g. GPC3).

(2) Generally, two genes in the proto-oncogenes group or suppressor genes group are positively related. But if one is proto-oncogene and another is suppressor one, they are negatively correlated.

(3) According the relationship between the HCC core genes (HAMP, MTs, GPC3) and the trace metals $(\mathrm{Cu}, \mathrm{Fe}, \mathrm{Zn})$ in human body, suitable zinc supplementation may reduce the risk of liver cancer.

\section{REFERENCES}

[1] Bodzin, A. S., \& Busuttil, R. W. (2015). Hepatocellular carcinoma: Advances in diagnosis, management, and long term outcome. World journal of hepatology, 7(9), 1157.

[2] Chinnaswamy, A., \& Srinivasan, R. (2016). Hybrid Feature Selection Using Correlation Coefficient and Particle Swarm Optimization on Microarray Gene Expression Data. In Innovations in BioInspired Computing and Applications(pp. 229-239). Springer International Publishing.

[3] Cao, Q., Imbeaud, S., Datta, S., \& Zucman-Rossi, J. (2015). Authors' response: virus-host interactions in HBV-related hepatocellular carcinoma: more to be revealed?. Gut, 64(5), 853-854.

[4] Chen, J., Wang, H., Wang, J., Huang, S., \& Zhang, W. (2015). STAT1 inhibits human hepatocellular carcinoma cell growth through induction of p53 and Fbxw7. Cancer cell international, 15(1), 1.

[5] Braicu, C., Maia, B. M., Berindan-Neagoe, I., \& Calin, G. A. (2015). MiRNA Expression Assays. In Genomic Applications in Pathology (pp. 45-70). Springer New York.

[6] Guichard, C., Amaddeo, G., Imbeaud, S., Ladeiro, Y., Pelletier, L., Maad, I. B., ... \& Clément, B. (2012). Integrated analysis of somatic mutations and focal copy-number changes identifies key genes and pathways in hepatocellular carcinoma. Nature genetics, 44(6), 694-698.

[7] Alexander, I. E., \& Russell, D. W. (2015). The potential of AAV-mediated gene targeting for gene and cell therapy applications. Current Stem Cell Reports, 1(1), 16-22.

[8] Gao, W., Tang, Z., Zhang, Y. F., Feng, M., Qian, M., Dimitrov, D. S., \& Ho, M. (2015). Immunotoxin targeting glypican-3 regresses liver cancer via dual inhibition of Wnt signalling and protein synthesis. Nature communications, 6.

[9] Sugam Sharma, Udoyara S Tim, Johnny Wong, Shashi Gadia, Subhash C Sharma. A Brief Review on Leading Big Data Models. Data Science Journal, 2014. 
Computer Applications: An International Journal (CAIJ), Vol.3, No.1, February 2016

[10] Sugam Sharma, Ritu Shandilya, Srikanta Patnaik, Ashok Mahapatra. Leading NoSQL Models for Handling Big Data: A Brief Review. International Journal of Business Information Systems Inderscience 2014.

[11] Sugam Sharma, U S Tim, Shashi Gadia, Ritu Shandilya, and P Sateesh. Classification and Comparison of Leading NoSQL Big Data Models. 2014. International Journal of Big Data Intelligence (IJBDI), Inderscience.

[12] Sugam Sharma, U S Tim, Shashi Gadia, and Johnny Wong.(2015).Proliferating Cloud Density through Big Data Ecosystem, Novel XCLOUDX Classification and Emergence of as-a-Service Era.

[13] Sugam Sharma. (2015). Evolution of as-a-Service Era in Cloud. Cornell University Library.

[14] Sugam Sharma, U S Tim, Shashi Gadia, and Johnny Wong.(2015).Growing Cloud Density \& as-aService Modality and OTH-Cloud Classification in IOT Era.

[15] Sugam Sharma.(2015).An Extended Classification and Comparison of NoSQL Big Data Models.

[16] Sugam Sharma. (2015). Expanded cloud plumes hiding Big Data ecosystem. Future Generation Computer Systems, Elsevier.

[17] Hu, J, and Gao, Z.(2012) "Modules identification in gene positive networks of hepatocellular carcinoma using Pearson agglomerative method and Pearson cohesion coupling modularity." Journal of Applied Mathematics 2012 .

[18] $\mathrm{Hu}, \mathrm{J}$, and Gao, Z (2012). "Distinction immune genes of hepatitis-induced heptatocellular carcinoma." Bioinformatics 28.24 : 3191-3194.

[19] Hu, J, Gao, Z, and Weisen Pan (2013). "Multiangle Social Network Recommendation Algorithms and Similarity Network Evaluation." Journal of Applied Mathematics 2013.

[20] $\mathrm{Hu}, \mathrm{J}$, and Gao, Z(2014). "Data-based core genes screening for Hepatocellular Carcinoma." Industrial Electronics and Applications (ICIEA), 2014 IEEE 9th Conference on. IEEE.

[21] Hu, J., Hu, B., Pan,W., Zhou X.,\& Gao, Z (2016). "Target Genes and Core Genes of Hepatocellular Carcinoma Using Gene Force Algorithm and Gene Community Network." International Journal of Innovative Science, Engineering \& Technology 3.1: Accepted and in press.

[22] Gaba, R. C., Groth, J. V., Parvinian, A., Guzman, G., \& Casadaban, L. C. (2015). Gene expression in hepatocellular carcinoma: pilot study of potential transarterial chemoembolization response biomarkers. Journal of Vascular and Interventional Radiology, 26(5), 723-732.

[23] Dongiovanni, P., Lanti, C., Gatti, S., Rametta, R., Recalcati, S., Maggioni, M., ... \& Valenti, L. (2015). High Fat Diet Subverts Hepatocellular Iron Uptake Determining Dysmetabolic Iron Overload. PloS one, 10(2), e0116855.

[24] Gozzelino, R., \& Arosio, P. (2015). The importance of iron in pathophysiologic conditions. Frontiers in pharmacology, 6.

[25] Nagaraju, G. P., Basha, R., Rajitha, B., Alese, O. B., Alam, A., Pattnaik, S., \& El-Rayes, B. (2016). Aquaporins: their role in gastrointestinal malignancies. Cancer Letters.

[26] Grüngreiff, K., Reinhold, D., \& Wedemeyer, H. (2016). The role of zinc in liver cirrhosis. Annals of Hepatology: Official Journal of the Mexican Association of Hepatology, 15(1).

[27] Reda, R., Abbas, A. A., Mohammed, M., El Fedawy, S. F., Ghareeb, H., El Kabarity, R. H., ... \& Zakaria, D. (2015). The Interplay between Zinc, Vitamin D and, IL-17 in Patients with Chronic Hepatitis C Liver Disease. Journal of Immunology Research.

[28] Xie, F., Jia, L., Lin, M., Shi, Y., Yin, J., Liu, Y., ... \& Meng, Q. (2015). ASPP2 attenuates triglycerides to protect against hepatocyte injury by reducing autophagy in a cell and mouse model of non-alcoholic fatty liver disease. Journal of cellular and molecular medicine, 19(1), 155-164.

[29] Cui, X., Li, Z., Gao, P. J., Gao, J., \& Zhu, J. Y. (2015). Prognostic value of glypican-3 in patients with HBV-associated hepatocellular carcinoma after liver transplantation. Hepatobiliary \& Pancreatic Diseases International,14(2), 157-163.

[30] Gopaul, K., A Shintre, S., \& A Koorbanally, N. (2015). A Review on the Synthesis and Anti-cancer Activity of 2-substituted Quinolines. Anti-Cancer Agents in Medicinal Chemistry (Formerly Current Medicinal Chemistry-Anti-Cancer Agents), 15(5), 631-646.

[31]Vela, G., Stark, P., Socha, M., Sauer, A. K., Hagmeyer, S., \& Grabrucker, A. M. (2015). Zinc in Gut-Brain Interaction in Autism and Neurological Disorders. Neural plasticity, 2015.

[32] Sun, Q., Zhong, W., Zhang, W., Sun, X., \& Zhou, Z. (2015). Subcellular Zinc Deficiency Links with ER and Mitochondria Cell Death Signaling in Alcoholic Liver Disease. The FASEB Journal, 29(1 Supplement), 1004-8. 


\section{Authors}

\section{Jinyu Hu, Postdoctoral Fellow, USA}

Jinyu Hu works in the Stanford University and Veterans Affairs Palo Alto Health Care System as a bioinformatics postdoctoral researcher. And she is a member in the Cipher Ground, whose job is to do some biology data analysis.

\section{Bin Hu, Postdoctoral Fellow, USA}

Bin $\mathrm{Hu}$ received his Ph.D. degree at Peking University and he is currently a postdoctoral fellow at Stanford University, his main research interests include molecular and biochemistry, immunology and translational medicine.

\section{Weisen Pan, Postdoctoral Fellow, USA}

Weisen Pan is a Post-doc in the Department of Computer Science and Engineering, The Ohio State University, Ohio, U.S. His research interests are in the areas of Deep Learning, Computer Vision and Bioinformatics.

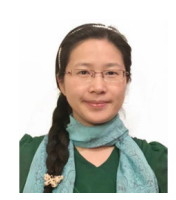

\section{Xiangtao Liu, Associate Research Scientist, USA}

Xiangtao Liu is an associate research scientist in the Dept. of Psychiatry of the University of Iowa. And in the Cipher Ground, he manages the genome science and technology, investment and quantify, and IT. His research interests involve statistical genetics, bioinformatics and numerical methods.

\section{Zhiwei Gao, Reader, UK}

Dr. Gao joined the University of Northumbria as Senior Lecturer in 2011, and was promoted to Reader in 2015. Dr. Gao is currently the Departmental PGR Programme Leader, and the Weekly Research Seminar Coordinator.
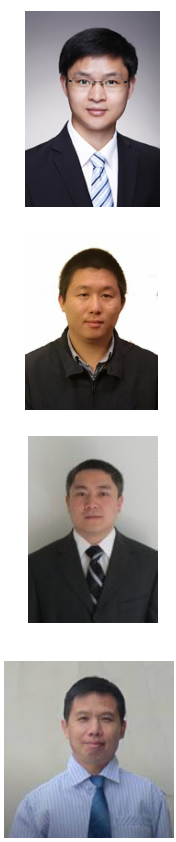